International Journal of Modern Physics B

Vol. 25, No. 28 (2011) 3691-3706

(C) World Scientific Publishing Company

DOI: $10.1142 /$ S0217979211102149

\title{
SUPERCONDUCTIVITY IN THE 2D ATTRACTIVE HUBBARD MODEL WITHIN A FUNCTIONAL FIELD-THEORETICAL RG
}

\author{
EBERTH CORREA \\ Instituto de Ciências Tecnológicas e Exatas, \\ Universidade Federal do Triângulo Mineiro, \\ 38064-200 Uberaba-MG, Brazil \\ eberthcorrea@gmail.com \\ HERMANN FREIRE \\ Instituto de Física, Universidade Federal de Goiás, \\ 74001-970 Goiânia-GO, Brazil \\ ALVARO FERRAZ \\ International Institute of Physics, \\ Universidade Federal do Rio Grande do Norte, \\ Caixa Postal 1641, 59072-970 Natal-RN, Brazil \\ Received 27 August 2010 \\ Revised 30 July 2011
}

\begin{abstract}
We apply the functional field-theoretical renormalization group methodology up to twoloop order to the 2D attractive Hubbard model for weak bare interaction. Since the momentum-resolved quasiparticle weight $Z_{\Lambda}(\mathbf{p})$ is always close to unity in the RG flow, higher-order quantum fluctuations do not affect crucially the low-energy dynamics in this case. As a result, we observe a $s$-wave singlet superconducting quasi-long-range order phase as a function of doping in good agreement with other numerical methods. To assess the role played by the fluctuations we compare the critical temperature $T_{c}$ in which the quasi-long-range superconducting order phase takes place within our approach with mean-field theory and quantum Monte Carlo results.
\end{abstract}

Keywords: Renormalization group; attractive Hubbard model; superconductivity.

\section{Introduction}

The attractive Hubbard model (HM) is a paradigmatic model to study the so-called BCS-BEC phase transition in strongly correlated systems. It generally describes an electron gas, in which the corresponding particles interact with each other locally via an attractive local interaction $U$. In recent days, this model has increased even further in importance. Thanks to ground-breaking experiments with ultracold gases trapped in optical lattices, ${ }^{1}$ this model can now be simulated and the corresponding 
interaction parameter can be tuned to strong coupling via Feshbach resonances. These experiments hold the important promise of simulating any given stronglycorrelated model in order to obtain exactly its ground state and the corresponding low-energy excitations. For instance, it could potentially settle the fundamental question regarding the possibility of existence of a $d$-wave superconducting (SC) phase in the two-dimensional (2D) repulsive HM, which is generally believed to be central to describe the cuprate superconductors.

On the theoretical side, several computational techniques ${ }^{2-4}$ such as quantum cluster approaches, exact diagonalization and quantum Monte Carlo (QMC) have been employed to probe both the attractive and repulsive HM. However, even those numerical methods which are used to investigate the low temperature properties produced essentially by quantum fluctuations — are plagued with some technical difficulties such as the fermionic minus sign problem in QMC simulations for the repulsive case, small cluster sizes in cluster approaches and other problems, which impose some restrictions in their implementation so far.

One possible way out is to resort to (semi-)analytical techniques such as the renormalization group (RG) approach..$^{5-18}$ In this work, we shall employ the socalled functional field-theoretical renormalization group (FFTRG) method. ${ }^{16,17}$ In $1 \mathrm{D}$, this approach reproduces some of the important features of the Luttinger liquid state when suitably combined with the Ward identities (WI) constrained by the underlying symmetries of the model in good agreement with bosonization and other quantum field-theoretical methods. ${ }^{19-23}$ However, in higher dimensions, the corresponding FFTRG equations become much more complex to be solved analytically and, for this reason, one has to resort necessarily to numerical methods. In earlier works, ${ }^{16,17,24,25}$ we pointed out the importance of self-energy corrections for the repulsive HM in 2D. These effects are typically of higher order and, therefore, one has to go beyond one-loop in the truncation scheme, before finally arriving at the FFTRG equations for the renormalized couplings.

In our previous work ${ }^{24}$ concerning the repulsive HM, we calculated the corresponding $\mathrm{RG}$ flow at two-loop order of the momentum-resolved quasiparticle weight $Z_{\Lambda}(\mathbf{p})$ and showed that the antiferromagnetic (AF) correlations present in the model tend to drive the system towards a metallic non-Fermi liquid (NFL) state with a truncated Fermi surface (FS) in the lightly doped regime. This phase presents itself in between an AF and a $d$-wave SC phase. This NFL state is characterized by a $Z_{\Lambda}(\mathbf{p})$ approaching zero in the anti-nodal regions and a small but finite weight around the nodal directions of the Brillouin zone. As the doping parameter is increased, the AF correlations become weaker due to the FS curvature effects. In contrast, the $d$-wave SC correlations become stronger and the competition between these two phases becomes more pronounced as we approach the quasi-long-ranged $d$-wave SC regime.

Another crucial test to the FFTRG equations is to check if they can also describe some of the important features of the attractive 2D Hubbard model (AHM). Studies of an extended version of this model have given significant contributions to the 
understanding of several properties of the AHM, in which a SC phase is stabilized at zero temperature with the addition of an intersite interaction even at half-filling. ${ }^{26}$ From a RG point of view, the onset of the SC phase can be studied by adding a small field in the corresponding channel to drive the system towards a continuous symmetry breaking with the RG flow. This provides a way to assess the reduction of the SC gap in the symmetry-broken phase with fluctuations if compared with standard mean-field results. ${ }^{27}$ In our implementation of the FFTRG equations up to two-loop order we will restrict our analysis only to the symmetric phase of the model, but some important features pointed out in Ref. 27 will be considered in our arguments. Therefore, our study will focus mainly on the analysis of the main instabilities of this model — signaling the onset of quasi-long-range order — with respect to specific response functions such as $s$-type charge density wave ( $s$-CDW), $d$-type charge density wave $(d$-CDW), the $s$-type spin density wave ( $s$-SDW), the $d$-type spin density wave $(d$-SDW), the $s$-wave singlet superconductivity ( $s$-SSC) and, finally, the $d$-wave singlet superconductivity ( $d$-SSC).

As opposed to the repulsive case, only $s$-wave superconductivity is manifest in the AHM. This is confirmed by several numerical schemes. ${ }^{28-38}$ Due to the strong renormalization of the so-called Umklapp scattering processes in our FFTRG equations, we will however limit our analysis to a maximum weak-coupling initial value of $|U|=2 t$. As it will become clear shortly, even in this weak coupling regime the renormalized couplings of the model flow to a strong coupling in the low-energy limit. As a result, they need to be conveniently stopped at an appropriate upper limit to avoid artificial divergences of the couplings considered in the model.

In this work, we investigate some possible electronic phases that emerge out of the renormalization of the coupling functions of the model in the low-energy limit. Our RG calculation confirms the existence of two leading instabilities in the AHM: charge density wave of $s$-type symmetry ( $s$-CDW) and $s$-wave singlet superconductivity ( $s$-SSC). In addition, the competition between these instabilities as a function of doping is also captured by our RG scheme. Indeed, as we will argue below, the FS curvature effects which are related to the electron density of the model, affect in a direct way the renormalization of the couplings. This generates correctly the $s$-SSC quasi-long-range order phase present in the model away from half-filling.

In Sec. 2 we give a brief guide to obtain the FFTRG equations within our scheme. In Sec. 3 we define the symmetries of the response functions relevant to our work. The details about the numerical approach and the results are shortly discussed in Sec. 4. Finally, we present our conclusions in Sec. 5.

\section{Field-Theoretical Renormalization Group Approach}

Our starting point is the HM given in momentum space by

$$
H=\sum_{\mathbf{k}, \sigma} \xi_{\mathbf{k}} \psi_{\mathbf{k} \sigma}^{\dagger} \psi_{\mathbf{k} \sigma}+\left(\frac{U}{N_{s}}\right) \sum_{\mathbf{p}, \mathbf{k}, \mathbf{q}} \psi_{\mathbf{p}+\mathbf{k}-\mathbf{q} \uparrow}^{\dagger} \psi_{\mathbf{q} \downarrow}^{\dagger} \psi_{\mathbf{k} \downarrow} \psi_{\mathbf{p} \uparrow},
$$


where $\psi_{\mathbf{k} \sigma}^{\dagger}$ and $\psi_{\mathbf{k} \sigma}$ are the fermionic creation and annihilation operators with momentum $\mathbf{k}$ and spin projection $\sigma, U<0$ is the local on-site attractive interaction and $N_{s}$ is the total number of lattice sites. As showed elsewhere, ${ }^{24}$ we can linearize the tight-binding energy dispersion $\xi_{\mathbf{k}}=-2 t\left[\cos \left(k_{x}\right)+\cos \left(k_{y}\right)\right]-\mu \approx$ $v_{F}(\mathbf{k}) \hat{\mathbf{n}} .\left(\mathbf{k}-\mathbf{k}_{F}(\mu)\right)$ with $v_{F}(\mathbf{k})=\left|\left(\left.\nabla_{\mathbf{k}} \xi_{\mathbf{k}}\right|_{\mathbf{k}=\mathbf{k}_{F}(\mu)}\right)\right|=2 t \sqrt{\sin ^{2} k_{x}+\sin ^{2} k_{y}}$. The Fermi momentum $\mathbf{k}_{F}(\mu)$ defines the noninteracting FS for a continuous chemical potential $\mu$ which is directly related to the density of particles, $\hat{\mathbf{n}}$ is a unit vector perpendicular to the FS and $t$ is the electronic hopping amplitude to nearest neighbors.

All the physical quantities are calculated perturbatively within the fieldtheoretical RG approach. One important point we wish to stress here is the fact that perturbative calculations for this model are in general plagued by divergences in the low-energy limit. As we will see shortly, the regularization of the model can be formally done by means of the Lagrangian formalism in which counterterms can be added to deal with these divergences in a consistent way order by order in perturbation theory. For this reason, instead of the Hamiltonian of the model (1) we will directly work with the following renormalized Lagrangian

$$
\begin{aligned}
L[\bar{\psi}, \psi]= & \sum_{\sigma} \int_{\mathbf{k}} Z_{\Lambda}(\mathbf{k}) \bar{\psi}_{\sigma}(\mathbf{k}, t)\left(i \partial_{t}+\xi_{\mathbf{k}}\right) \psi_{\sigma}(\mathbf{k}, t) \\
& +\sum_{\sigma, \sigma^{\prime}} \int_{\mathbf{p}_{\mathbf{1}}} \int_{\mathbf{p}_{\mathbf{2}}} \int_{\mathbf{p}_{\mathbf{3}}}\left[\prod_{i=1}^{4} Z_{\Lambda}\left(\mathbf{p}_{\mathbf{i}}\right)\right]^{1 / 2} g_{B}\left(\mathbf{p}_{\mathbf{1}}, \mathbf{p}_{\mathbf{2}}, \mathbf{p}_{\mathbf{3}}\right) \\
& \times \bar{\psi}_{\sigma}\left(\mathbf{p}_{\mathbf{1}}+\mathbf{p}_{\mathbf{2}}-\mathbf{p}_{\mathbf{3}}, t\right) \bar{\psi}_{\sigma^{\prime}}\left(\mathbf{p}_{\mathbf{3}}, t\right) \psi_{\sigma^{\prime}}\left(\mathbf{p}_{\mathbf{2}}, t\right) \psi_{\sigma}\left(\mathbf{p}_{\mathbf{1}}, t\right),
\end{aligned}
$$

where $\int_{\mathbf{k}}=\int\left(d^{2} \mathbf{k} /(2 \pi)^{2}\right)$ and $g_{B}$ are the bare couplings related to the renormalized ones by

$$
g_{B}=\left[\prod_{i=1}^{4} Z_{\Lambda}^{-\frac{1}{2}}\left(\mathbf{p}_{\mathbf{i}}\right)\right]\left[g_{R}\left(\mathbf{p}_{\mathbf{1}}, \mathbf{p}_{\mathbf{2}}, \mathbf{p}_{\mathbf{3}} ; \Lambda\right)+\Delta g_{R}\left(\mathbf{p}_{\mathbf{1}}, \mathbf{p}_{\mathbf{2}}, \mathbf{p}_{\mathbf{3}} ; \Lambda\right)\right],
$$

with $Z_{\Lambda}(\mathbf{p})$ being the well-known quasiparticle weight which depends on the momentum at the FS and on the RG scale $\Lambda$. Equation (2) defines our quantum field theory which is also regularized in the ultraviolet limit by restricting the energies to $\left|p_{0}\right| \leq \Lambda_{0}$ with $\Lambda_{0}=4 t$ being half of the energy bandwidth.

The physical properties of this model at $T=0$ can be computed from the coherent-state Grassmann representation of the partition function

$$
\mathcal{Z}=\int \mathcal{D}[\bar{\psi}, \psi] e^{i \int d t L[\bar{\psi}, \psi]} .
$$

In what follows, it is worth mentioning that two logarithmically divergent bubbles frequently appear within perturbation theory: the particle-particle (PP) and particle-hole $(\mathrm{PH})$ bubbles. They are displayed in Fig. 1. By using the Feynman 

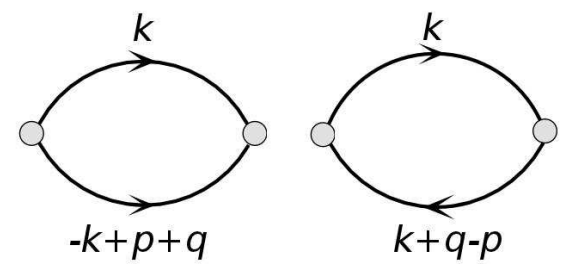

Fig. 1. The PP and $\mathrm{PH}$ bubbles respectively.

rules for diagrammatic calculations we obtain that these bubbles are given approximately by

$$
\begin{aligned}
& \Pi_{\mathrm{PP}} \approx\left(\frac{1}{v_{F}(\mathbf{k})+v_{F}(-\mathbf{k}+\mathbf{p}+\mathbf{q})}\right) \ln \left(\frac{\Lambda_{0}}{\Lambda}\right), \\
& \Pi_{\mathrm{PH}} \approx-\left(\frac{1}{v_{F}(\mathbf{k})+v_{F}(\mathbf{k}+\mathbf{p}-\mathbf{q})}\right) \ln \left(\frac{\Lambda_{0}}{\Lambda+2|\mu| F(\mathbf{k}, \mathbf{p}, \mathbf{q})}\right),
\end{aligned}
$$

where $\Pi_{\mathrm{PP}}$ refers to the $\mathrm{PP}$ bubble, $\Pi_{\mathrm{PH}}$ represents the $\mathrm{PH}$ contribution, and $F(\mathbf{p}, \mathbf{k}, \mathbf{q})=v_{F}(\mathbf{k}+\mathbf{q}-\mathbf{p}) / v_{F}(\mathbf{k})$. By integrating the perpendicular degrees of freedom of the model only the momenta along the FS (or parallel to the FS) remains in the low-energy limit. For this reason, from here on all momenta appearing in the our FFTRG equations are always projected along the noninteracting FS of the model.

Notice the factor in the denominator of the logarithm due to the $\mathrm{PH}$ bubble. This term is responsible for the strength of the $\mathrm{PH}$ contributions in the FFTRG equations. If the energy scale $\Lambda$ is such that this bubble takes large values in the infrared limit, both $\mathrm{PP}$ and $\mathrm{PH}$ bubbles will compete with each other on equal footing. This is more evident insofar as we get closer of half-filling. As a physical result, the density waves are competing with pair formation with some relative weight, which is associated with the curvature of the FS. On the other hand, the FFTRG equations are strongly affected by the van Hove points. In fact, the Fermi velocities along the FS, appearing in the denominator of the Eq. 5, can be very small at the corners of the FS close to half-filling.

Now, we move on to the FFTRG treatment of the interactions. Since we go up to two-loop order in our RG approach, the renormalized counterterms associated with the regularization of the vertex corrections of the model are $^{17}$

$$
\Delta g_{R}=\Delta g_{R}^{1 \text { loop }}\left(\mathbf{p}_{\mathbf{1}}, \mathbf{p}_{\mathbf{2}}, \mathbf{p}_{\mathbf{3}} ; \Lambda\right)+\Delta g_{R}^{2 \text { loops }}\left(\mathbf{p}_{\mathbf{1}}, \mathbf{p}_{\mathbf{2}}, \mathbf{p}_{\mathbf{3}} ; \Lambda\right),
$$

which must be properly added to the Lagrangian to regularize the infrared singularities as mentioned before.

The marginally relevant scattering processes are displayed in Fig. 2. In this work, we are only considering interactions which produce the already mentioned logarithmic singularities when one scales the loop integrals towards the FS. As a result, by power counting arguments, the momenta dependence normal to the FS of 

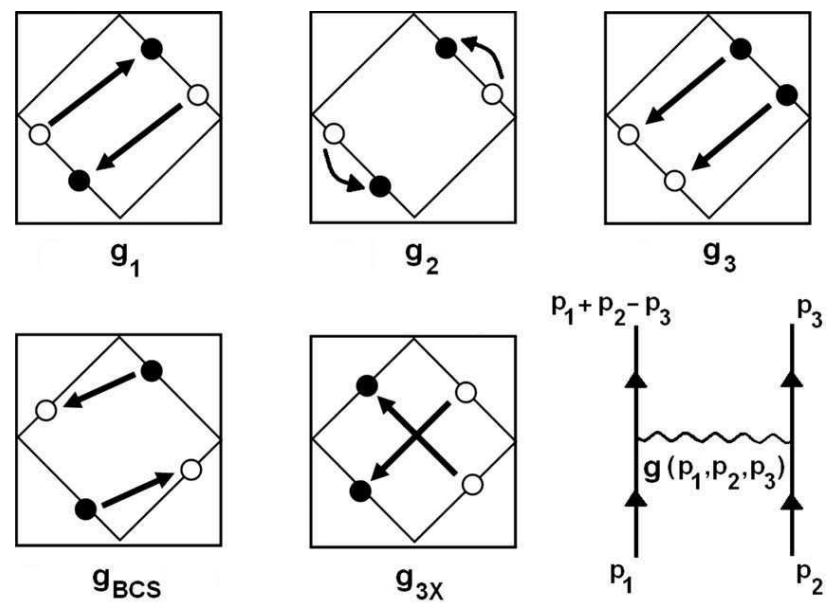

Fig. 2. The g-ology parametrization for the marginally relevant interaction processes of the 2D HM, using as a reference the corresponding noninteracting FS at half-filling for simplicity.

the couplings is irrelevant. ${ }^{11}$ The scattering processes present in our model are: the backscattering $g_{1}$, the forward scattering $g_{2}$, the Umklapp processes $g_{3}$ and $g_{3 x}$ and, finally, the BCS type interaction $g_{\mathrm{BCS}}$. For simplicity, we neglect the contributions arising from the scattering process associated with the so-called $g_{4}$ couplings. We expect that this approximation will not change our results qualitatively. The next step in the FFTRG treatment is to calculate the quasiparticle weight which plays an important role in the repulsive HM. ${ }^{16,17,24,25}$ It is worthwhile emphasizing that in one-loop approximation the self-energy produces no contribution to $Z_{\Lambda}$. The first nonzero contribution shows up in two-loop order and is produced by the so-called sunset diagram displayed in Fig. 3.

As usual, to derive the flow equations in the FFTRG we must select an appropriate prescription for the renormalized correlation functions at the FS. Since $Z_{\Lambda}$ is directly associated with the single-particle Green's function $G_{R}\left(\mathbf{p}, p_{0} ; \Lambda\right)$, we choose $G_{R}\left(\mathbf{p}, p_{0}=\Lambda\right)=1 / \Lambda$. Following the procedure detailed in our Ref. 17 we arrive at the flow equation

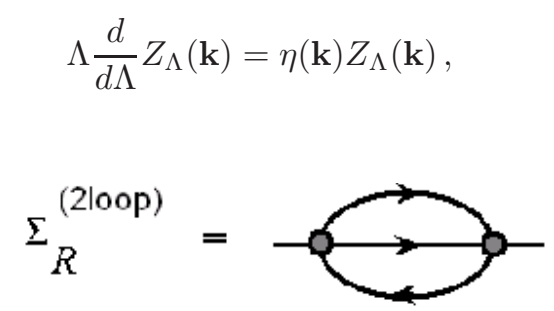

Fig. 3. The self-energy diagrams up to two-loop order. The one-loop term is the Hartree diagram and the two-loop contribution is the sunset diagram. 
which defines the anomalous dimension $\eta$ given by ${ }^{17}$

$$
\begin{aligned}
\eta(\mathbf{p})= & \frac{1}{8 \pi^{4}} \int d \mathbf{k} \int d \mathbf{q}\left[\frac{\Lambda}{\Lambda+2|\mu| F(\mathbf{p}, \mathbf{k}, \mathbf{q})}\right] \\
& \times\left[2 g_{1 R}(\mathbf{p}, \mathbf{k}+\mathbf{q}-\mathbf{p}, \mathbf{k}) g_{1 R}(\mathbf{k}, \mathbf{q}, \mathbf{p})\right. \\
& +2 g_{2 R}(\mathbf{p}, \mathbf{k}+\mathbf{q}-\mathbf{p}, \mathbf{q}) g_{2 R}(\mathbf{k}, \mathbf{q}, \mathbf{k}+\mathbf{q}-\mathbf{p}) \\
& -g_{1 R}(\mathbf{p}, \mathbf{k}+\mathbf{q}-\mathbf{p}, \mathbf{k}) g_{2 R}(\mathbf{k}, \mathbf{q}, \mathbf{k}+\mathbf{q}-\mathbf{p}) \\
& -g_{2 R}(\mathbf{p}, \mathbf{k}+\mathbf{q}-\mathbf{p}, \mathbf{q}) g_{1 R}(\mathbf{k}, \mathbf{q}, \mathbf{p})+2 g_{3 R}(\mathbf{k}, \mathbf{p}, \mathbf{q}) \\
& \left.\times g_{3 R}(\mathbf{k}, \mathbf{p}, \mathbf{q})-g_{3 R}(\mathbf{k}, \mathbf{p}, \mathbf{q}) g_{3 R}(\mathbf{p}, \mathbf{k}, \mathbf{q})\right] \\
& \times\left(\frac{1}{v_{F}(\mathbf{k})+v_{F}(\mathbf{q})}\right)\left(\frac{1}{v_{F}(\mathbf{q})+v_{F}(\mathbf{k}+\mathbf{q}-\mathbf{p})}\right),
\end{aligned}
$$

The FFTRG flow equations for the renormalized coupling functions are given by

$$
\begin{aligned}
\Lambda \frac{d}{d \Lambda} g_{i R}\left(\mathbf{p}_{\mathbf{1}}, \mathbf{p}_{\mathbf{2}}, \mathbf{p}_{\mathbf{3}}\right)= & \frac{1}{2} \sum_{i=1}^{4} \eta\left(\mathbf{p}_{\mathbf{i}}\right) g_{i R}\left(\mathbf{p}_{\mathbf{1}}, \mathbf{p}_{\mathbf{2}}, \mathbf{p}_{\mathbf{3}}\right) \\
& -\Lambda \frac{d}{d \Lambda} \Delta g_{i R}^{1 \text { loop }}\left(\mathbf{p}_{\mathbf{1}}, \mathbf{p}_{\mathbf{2}}, \mathbf{p}_{\mathbf{3}}\right) \\
& -\Lambda \frac{d}{d \Lambda} \Delta g_{i R}^{2 \text { loops }}\left(\mathbf{p}_{\mathbf{1}}, \mathbf{p}_{\mathbf{2}}, \mathbf{p}_{\mathbf{3}}\right),
\end{aligned}
$$

where $i=1,2,3,3 X$, and BCS. We illustrate in Fig. 4 some of the Feynman diagrams which are automatically taken into account by our scheme. ${ }^{17}$ We define next the response functions which are important for discussing the low-energy properties of the AHM.
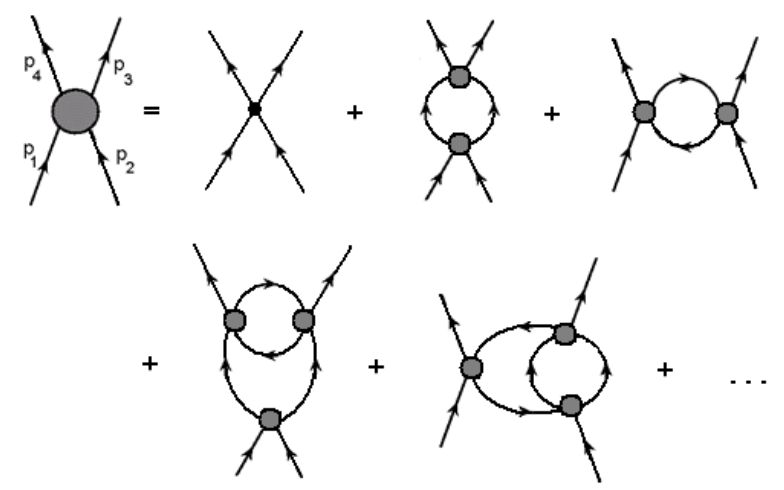

Fig. 4. Some Feynman diagrams showing the vertex corrections up to two-loop order. 


\section{Response Functions}

Once the RG equations for the couplings and the quasiparticle weight are established, we must introduce next both superconducting and density-wave instability sources in our renormalized Lagrangian. We add therefore the following term

$$
\begin{aligned}
L_{\mathrm{ext}}= & \sum_{\alpha \beta} \int_{\mathbf{q}, \mathbf{p}}\left[Z_{\Lambda}^{1 / 2}(\mathbf{p}) Z_{\Lambda}^{1 / 2}(\mathbf{q}-\mathbf{p}) \gamma_{B, \mathrm{SC}}^{\alpha \beta}(\mathbf{p}, \mathbf{q})\right. \\
& \times h_{\mathrm{SC}}(\mathbf{q}) \bar{\psi}_{\alpha}(\mathbf{p}, t) \bar{\psi}_{\beta}(-\mathbf{p}+\mathbf{q}, t) \\
& +Z_{\Lambda}^{1 / 2}(\mathbf{p}) Z_{\Lambda}^{1 / 2}(\mathbf{p}+\mathbf{q}) \gamma_{B, \mathrm{DW}}^{\alpha \beta}(\mathbf{p}, \mathbf{q}) \\
& \left.\times h_{\mathrm{DW}}(\mathbf{q}) \bar{\psi}_{\alpha}(\mathbf{p}+\mathbf{q}, t) \psi_{\beta}(\mathbf{p}, t)+\text { h.c. }\right],
\end{aligned}
$$

where $h_{\mathrm{SC}}(\mathbf{q})$ and $h_{\mathrm{DW}}(\mathbf{q})$ are external fields that must go to zero at the end of the calculations. ${ }^{24,25} \gamma_{B, S C}^{\alpha \beta}(\mathbf{p}, \mathbf{q})$ and $\gamma_{B, \mathrm{DW}}^{\alpha \beta}(\mathbf{p}, \mathbf{q})$ are the bare response vertices for probing pairing and density-wave instabilities, respectively. These terms will generate new Feynman diagrams which are also singular in the infrared limit. Some of those diagrams are displayed in Fig. 5. Following our FFTRG treatment for the response functions we get $^{24}$

$$
\begin{aligned}
\Lambda \frac{d}{d \Lambda} \gamma_{R, \mathrm{SC}}^{\alpha \beta}(\mathbf{p}, \mathbf{q})= & \frac{1}{2}[\eta(\mathbf{p})+\eta(-\mathbf{p}+\mathbf{q})] \gamma_{R, \mathrm{SC}}^{\alpha \beta}(\mathbf{p}, \mathbf{q}) \\
& -\Lambda \frac{d}{d \Lambda} \Delta \gamma_{R, \mathrm{SC}}^{\alpha \beta}(\mathbf{p}, \mathbf{q}) \\
\Lambda \frac{d}{d \Lambda} \gamma_{R, \mathrm{DW}}^{\alpha \beta}(\mathbf{p}, \mathbf{q})= & \frac{1}{2}[\eta(\mathbf{p}+\mathbf{q})+\eta(\mathbf{p})] \gamma_{R, \mathrm{DW}}^{\alpha \beta}(\mathbf{p}, \mathbf{q}) \\
& -\Lambda \frac{d}{d \Lambda} \Delta \gamma_{R, \mathrm{DW}}^{\alpha \beta}(\mathbf{p}, \mathbf{q}),
\end{aligned}
$$

after integrating the perpendicular degrees of freedom. As mentioned above, all the momenta must be taken along the curve delimited by the FS.

We are interested here only in some spin configurations of Eq. (10). They are obtained if we appropriately symmetrize $\gamma_{\mathrm{SC}}^{\alpha \beta}(\mathbf{p}, \mathbf{q})$ and $\gamma_{\mathrm{DW}}^{\alpha \beta}(\mathbf{p}, \mathbf{q})$ with respect to their spin indices

$$
\begin{aligned}
\gamma_{\mathrm{SSC}}(\mathbf{p}, \mathbf{q}) & =\gamma_{\mathrm{SC}}^{\uparrow \downarrow}(\mathbf{p}, \mathbf{q})-\gamma_{\mathrm{SC}}^{\downarrow \uparrow}(\mathbf{p}, \mathbf{q}), \\
\gamma_{\mathrm{SDW}}(\mathbf{p}, \mathbf{q}) & =\gamma_{\mathrm{DW}}^{\uparrow \uparrow}(\mathbf{p}, \mathbf{q})-\gamma_{\mathrm{DW}}^{\downarrow \downarrow}(\mathbf{p}, \mathbf{q}), \\
\gamma_{\mathrm{CDW}}(\mathbf{p}, \mathbf{q}) & =\gamma_{\mathrm{DW}}^{\uparrow \uparrow}(\mathbf{p}, \mathbf{q})+\gamma_{\mathrm{DW}}^{\downarrow \downarrow}(\mathbf{p}, \mathbf{q}),
\end{aligned}
$$

where SSC refers to singlet superconductivity and SDW and CDW relate to charge and spin density waves, respectively. Notice that this symmetrization does not affect the RG treatment and one can use Eq. (11) for the renormalized symmetrized response functions. The symmetry of the response functions is determined by these 

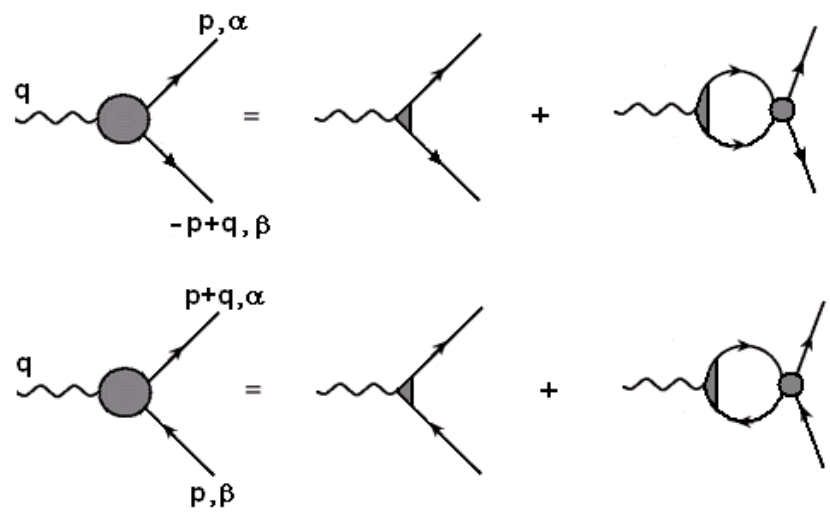

Fig. 5. The Feynman diagrams for the three-legged response vertices associated with superconducting and density-wave orders.

initial conditions of these RG equations and are given by

$$
\begin{aligned}
& \gamma_{i R}\left(\mathbf{p}, \mathbf{q} ; \Lambda=\Lambda_{0}\right)=1 \quad(s \text {-wave }) \\
& \gamma_{i R}\left(\mathbf{p}, \mathbf{q} ; \Lambda=\Lambda_{0}\right)=\frac{1}{\sqrt{2}}\left(\cos p_{x}-\cos p_{y}\right) \quad\left(d_{\left.x^{2}-y^{2} \text {-wave }\right),}\right.
\end{aligned}
$$

where $i=$ SSC, CDW and SDW. With the help of the Feynman diagrams displayed in Fig. 6 we finally obtain ${ }^{24}$

$$
\begin{aligned}
\Lambda \frac{d}{d \Lambda} \chi_{R, \mathrm{SC}}(\mathbf{q}=0 ; \Lambda)= & -\int \frac{d \mathbf{p}}{v_{F}(\mathbf{p})}\left[\gamma_{R, \mathrm{SC}}(\mathbf{p},-\mathbf{p} ; \Lambda)\right]^{2}, \\
\Lambda \frac{d}{d \Lambda} \chi_{R, \mathrm{DW}}(\mathbf{q}=\mathbf{Q} ; \Lambda)= & -\left(\frac{\Lambda}{\Lambda+2|\mu|}\right) \int \frac{d \mathbf{p}}{v_{F}(\mathbf{p})} \\
& \times\left[\gamma_{R, D W}(\mathbf{p}+\mathbf{Q}, \mathbf{p} ; \Lambda)\right]^{2},
\end{aligned}
$$

where we now write SC for singlet superconductivity and DW for charge and spin density wave susceptibilities to avoid overloading the notation. The vector $\mathbf{Q}$ is the perfect nesting wave vector.

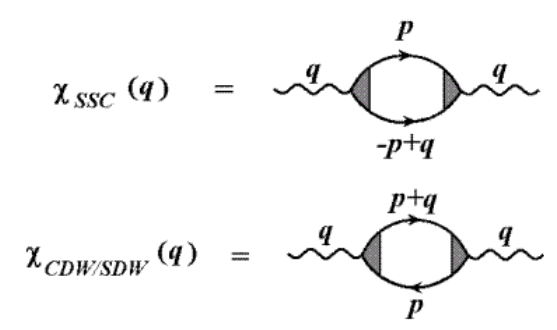

Fig. 6. Feynman diagrams for the susceptibilities with singlet superconductivity and charge and spin density wave orders. 


\section{Numerical Results}

Here we follow the same numerical procedure detailed in our previous RG works. ${ }^{17,25}$ All the integrals are calculated considering $N=32$ FS patches. Despite this limited number of points, the distance between two different points at the FS is such that the integral step necessary for the calculations is at most 0.5 at half-filling. Once the integral step decreases with doping, as displayed in Fig. 7, we get a good convergence for all numerical results of the RG equations.

In the left panel of Fig. 8 we display the results for the flow of the couplings calculated by means of the Eq. (9) versus $\Lambda / 4 t$ and density $n \approx 0.833$. Although the doping is introduced in the HM via the chemical potential, we use the density

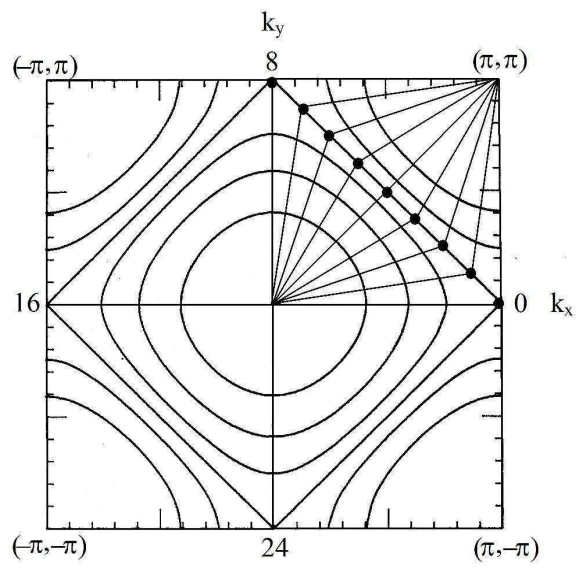

Fig. 7. The set of discrete points on the FS in the first quadrant. The numbers $0,8,16,24$ represent the antinodal directions, and the nodal points are the middle points between them.
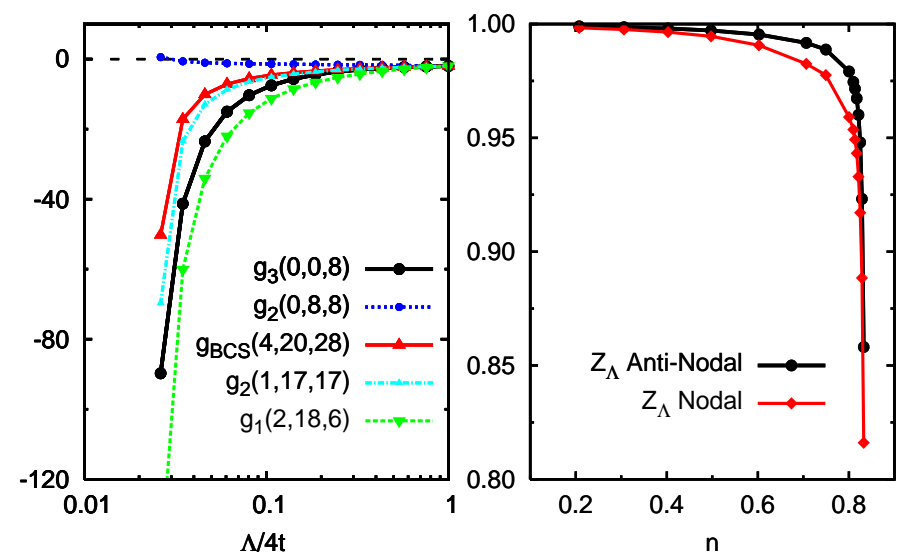

Fig. 8. (Color online). Left panel: The two-loop RG flow of the renormalized couplings (in units of $t$ ) for initial interaction $U=-2 t$ and density $n \approx 0.833$. Right panel: the quasiparticle weight $Z_{\Lambda}$ in the nodal and anti-nodal regions and same density. 
of particles $n$ to plot all the graphs displayed here in order to establish a direct comparison with other well-known works. ${ }^{28-40}$

As an initial condition for the couplings we choose $U=-2 t$. This choice is the maximum value we can assign for the bare interaction to solve numerically the FFTRG equations. This is directly related to the Umklapp processes, specially those associated with van Hove points. They renormalize very rapidly flowing to a strong coupling regime for all electronic densities. This behavior can be clearly seen in Fig. 8. To keep control of the flow of the FFTRG equations we fix an upper limit for the absolute value of the most pronounced one, i.e., $g_{\max }=200$. When this upper limit is reached, the flow of the renormalized couplings is immediately stopped.

In the right panel of Fig. 8, we display as an example the RG flow of the quasiparticle weight $Z$ for density $n \approx 0.833$ away from half-filling. In this regime, as can be seen from this figure, $Z$ is only very weakly renormalized. This implies that the metallic state of this model at this weak-coupling value indeed conforms to a Landau-Fermi liquid regime. This result is also in good agreement with dynamical mean-field theory (DMFT) calculations. ${ }^{34-36}$ However, although the renormalization of $Z$ is weak, its main contribution is to add correlations to the physical system. This can only be done consistently in an implementation of the RG scheme at two loops (as shown here in this work) or beyond. As a result, the pair formation in the present case is now favored, for the $\mathrm{PH}$ fluctuations are less divergent. ${ }^{5,25}$ Therefore, the main effect of the correlations into the RG equations is manifest by means of the critical energy scale in which the quasi-long-range order takes place. By contrast, another important point we wish to emphasize here is that the aforementioned scenario is very different from the corresponding model in the repulsive regime. In the repulsive case, the strong renormalization of $Z$ close to half-filling reveals that the $\mathrm{PH}$ fluctuations close to the Fermi surface act against pairing tendencies suggesting the formation of a non-Fermi liquid state in the vicinity of half-filling. ${ }^{24}$

Next, we move on to the results for the susceptibilities displayed in Fig. 9. By using the symmetries mentioned in the last section we can define the $s$-type charge density wave $(s$-CDW), $d$-type charge density wave $(d$-CDW), the $s$-type spin density wave $(s$-SDW), the $d$-type spin density wave $(d$-SDW), the $s$-wave singlet superconductivity ( $s$-SSC) and, finally, the $d$-wave singlet superconductivity ( $d$-SSC). As one can see from this figure, there is an energy scale in which the $s$-wave singlet superconductivity overcomes the charge density wave with $s$-symmetry signaling a crossover in the physical system. On the other hand, the other symmetries do not even reach the unity value as shown in the right panel of Fig. 9. For densities below 0.833 the $s$-SSC susceptibility always dominates the flow if compared with the flow of the $s$-CDW susceptibility. To illustrate this point, we plot the values of the two most pronounced susceptibilities when the upper limit for the renormalized couplings is reached in Fig. 10. When the particle density is decreased there is a strong suppression of the $s$-CDW susceptibility. Varying this density $n \approx 0.82-0.84$, the leading $s$-CDW fluctuations become weaker down to the critical regime in which 

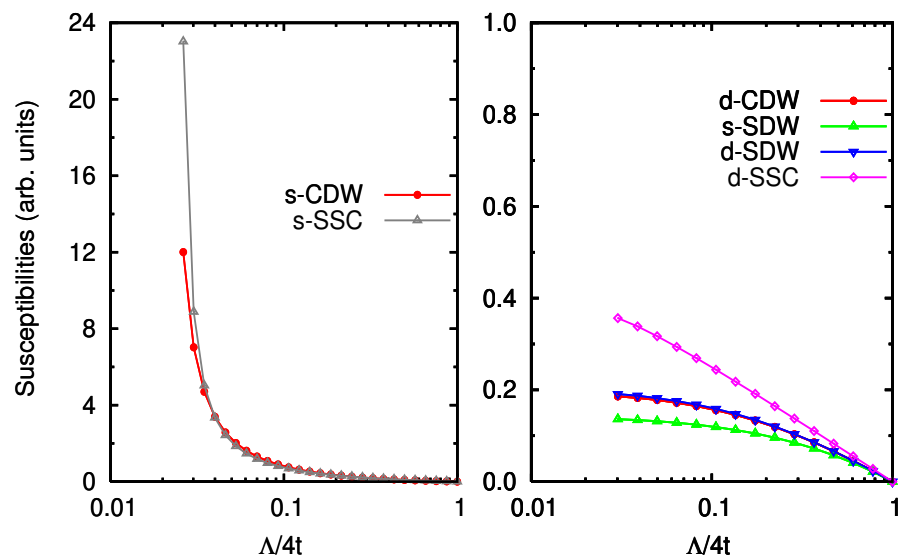

Fig. 9. (Color online). Left panel: The most pronounced $s$-CDW and $s$-SSC susceptibilities versus $\Lambda / 4 t$ for initial interaction $U=-2 t$ and density $n \approx 0.833$. Right panel: The renormalized susceptibilities versus $\Lambda / 4 t$ for initial interaction $U=-2 t$ and density $n \approx 0.833$.

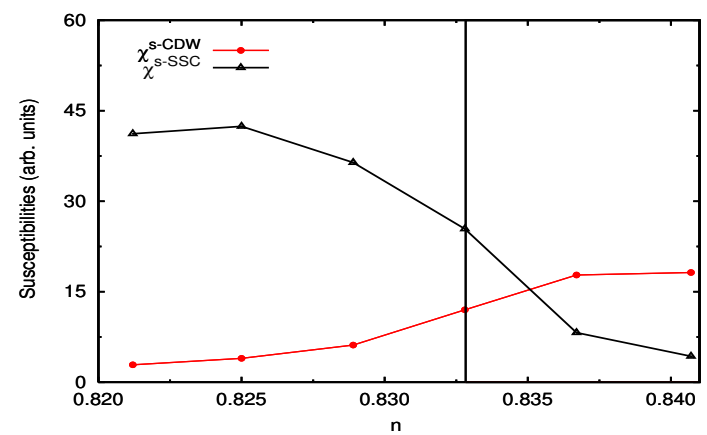

Fig. 10. (Color online). The $s$-CDW and $s$-SSC susceptibilities versus the density $n$. The lines are only a guide for the eyes.

the $s$-SSC susceptibility starts to compete on equal footing. Then at $n \approx 0.833$ a crossover occurs when the $s$-SSC correlations become the leading contribution during all the RG process for smaller densities.

This is a direct consequence of the renormalization of the coupling functions as displayed in the Fig. 11. In spite of the strong coupling behavior of some interaction processes, the majority of these couplings remain in a weak-to-moderate regime. The RG equations renormalize the backscattering and forward processes which in turn strengthen the BCS couplings as the doping is increased. The reason for this is rather simple: there are several Feynman diagrams which mix $g_{1 R}, g_{2 R}$ and $g_{3 R}$ processes in the backscattering and forward channels, specially at two-loop order. Similarly, several other diagrams mix $g_{1 R}, g_{2 R}$ and $g_{\mathrm{BCS}}^{R}$ in the BCS channel producing this kind of behavior. Thus, an enhancement in $g_{1 R}$ and $g_{2 R}$ due to a strong renormalization of $g_{3 R}$ also implies an enhancement of $g_{\mathrm{BCS}}^{R}$ due to both $g_{1 R}$ 


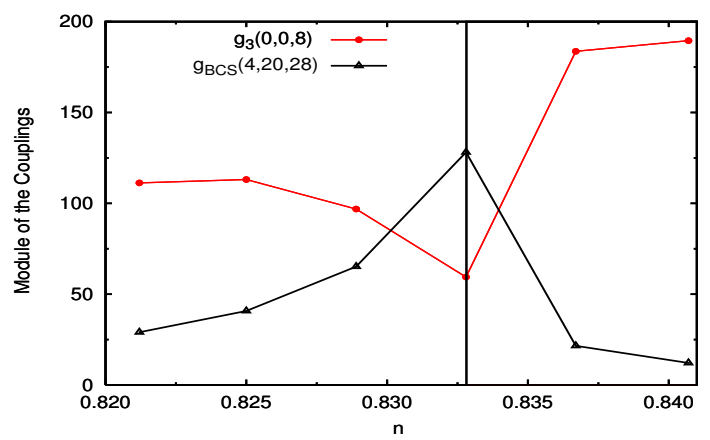

Fig. 11. (Color online). Two renormalized couplings of $g_{\mathrm{BCS}}$ and $g_{3}$ type versus the density $n$. The lines are only a guide for the eyes.

and $g_{2 R}$. In other words, the enhancement in the BCS channel favors the pairing of particles with total momentum equal to zero as required by processes in this channel. However, near half-filling the renormalization of $g_{3 R}$ is even stronger and the flow must be stopped before $g_{1 R}, g_{2 R}$ and $g_{\mathrm{BCS}}^{R}$ begin to renormalize themselves. Only at larger doping the renormalization of $g_{3 R}$ becomes weaker and these couplings renormalize appreciably driving the physical system to a quasi-long-range $\mathrm{SC}$ order as shown in Fig. 11. Since the doping is directly related to the curvature of the FS, we can safely conclude that the topology of the FS has dramatic consequences on the physical behavior of the system. The flatness of the FS can be related directly with the enhancement of the Umklapp processes which are very important for the charge density waves in the model. In contrast, the bigger the curvature the more important becomes the BCS processes driving the system to the $s$-SSC quasi-long-range order phase.

On the other hand, QMC calculations ${ }^{28-33}$ showed that away from half-filling the $s$-CDW correlations are suppressed and, at finite temperatures, a KosterlitzThouless (KT) phase transition with algebraic decay of the SC correlations takes place. This means that at any filling with $n \neq 1$ the SC phase is the dominant instability below this temperature. Due to the weak renormalization of the quasiparticle weight $Z$ together with the bare weak coupling regime it turns out that the BCS mean-field theory is a good approximation. ${ }^{40}$ Thus, the critical energy scale determined by the complete divergence of some renormalized couplings and the leading susceptibility can be assigned to the critical temperature $T_{c}$ for the onset of the quasi-long-range order phase. ${ }^{5}$ However, other calculations - in which fluctuations are added at one-loop RG level — showed that the SC gap is reduced if compared with that calculated via mean-field theory. ${ }^{27}$ This effect becomes more evident as the modulus of the bare interaction $U$ increases. This effect is in qualitative agreement with the results obtained here.

Although we are using an upper limit for the absolute value of the most pronounced renormalized coupling, we can estimate the critical energy scale $\Lambda_{c}$ associated with the complete divergence of some couplings and the leading $s$-SSC 


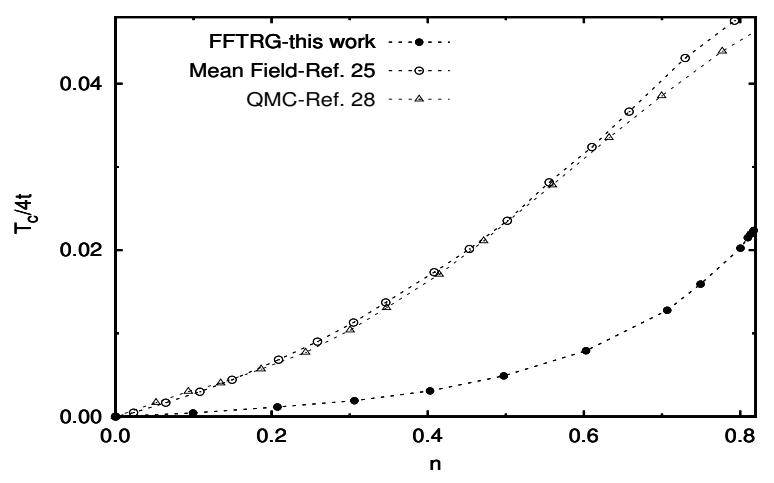

Fig. 12. The critical temperature versus the electron density $n$ for the attractive Hubbard model.

susceptibility. To this end, we check for infinite slopes on the most pronounced renormalized couplings and susceptibilities, which occur at $\Lambda_{c} / 4 t \approx 0.026$ for this particular density. If we suppose that it is also possible to associate this RG scale $\Lambda_{c}$ with an effective critical temperature scale $T_{c}$, this could in principle provide approximate estimates of the critical temperatures for the SC phase transition displayed by this model obtained within our two-loop RG scheme. By varying the density from $0.833-0$ and proceeding as mentioned above we plot the critical temperature $T_{c} / 4 t$ versus the electron density $n$ in Fig. 12 .

We can assess the role played by the fluctuations through the critical temperature for the onset of the quasi-long-range order SC phase as displayed in Fig. 12. They are clearly smaller than those obtained by mean-field and QMC calculations $^{26,28-33}$ due to the fluctuations added by the two-loop FFTRG equations in agreement with our expectations. As pointed out in other studies, ${ }^{41}$ the effect of including second order correlations by means of the self-energy is a reduction of $T_{c}$. This is due to the fact that the two-loop self-energy diagrams contribute with an opposite sign to the FFTRG equations for the interactions in which the divergences are retarded if compared with one-loop calculations. ${ }^{25}$ In addition, the complete divergence of some of the susceptibilities seems to occur in a rate slower than those for the couplings. To get an improvement for the temperatures at any density we should go to the finite temperature formalism for the FFTRG equations in which the correlation functions are those at a given temperature in the $\Lambda \rightarrow 0$ limit, but this is out of the scope of this work.

\section{Summary}

We have applied the FFTRG methodology to study the dominant instabilities in the AHM. By considering a weak bare interacting value of $|U|=2 t$, we have analyzed the competition of some susceptibilities as a function of density and the RG scale $\Lambda$. As a result, we have found a crossover region in the vicinity of $n \approx 0.833$. The physical system suggests two leading susceptibilities, which are directly related to 
both charge density wave $(s$-CDW) of $s$-type symmetry and $s$-wave singlet SC $(s-\mathrm{SSC})$ instabilities.

As we have seen, the RG flow of the marginally relevant scattering processes drives the system to the $s$-SSC quasi-long-range order phase for densities $n \lesssim 0.833$. The two-loop order diagrams which mix $g_{1 R}$ and $g_{3 R}$ and $g_{2 R}$ and $g_{3 R}$ become weaker when the density is decreased. Since most of the one-loop diagrams mix only $g_{1 R}, g_{2 R}$ and $g_{\mathrm{BCS}}$ this produces an enhancement in their corresponding correlation functions. Indeed, when the density is decreased, the FS curvature plays an important role. The Umklapp $g_{3 R}$ scattering processes are directly affected by the FS curvature in such a way that the greater the curvature the weaker theses processes renormalize the BCS channel as illustrated in Fig. 11.

The fact that the momentum-resolved $Z_{\Lambda}(\mathbf{p})$ does not renormalize significantly both near and far from half-filling suggests the existence of a coherent FS with quasiparticle excitations for the range of parameters studied here. As a result, for this weak value of $U$ and in all density regions considered here the metallic state conforms to a Landau-Fermi liquid description in agreement with DMFT studies. ${ }^{34-36}$ As a result, the critical temperature associated with this critical energy scale can be interpreted as a mean-field-like temperature. We used this argument to plot the critical temperature versus the density which were compared with mean-field (MF) and QMC calculations. ${ }^{26,28-33}$ They are clearly smaller than those calculated via $\mathrm{MF}$ and QMC due to the addition of fluctuations in the two-loop implementation of the FFTRG equations in agreement with our expectations.

In summary, the FS curvature is an important ingredient in order to obtain a better understanding of the driving mechanism of the superconductivity in the framework of the RG method. The interaction processes associated with any given model renormalize themselves in different ways depending on the initial topology of the FS. Since the quasiparticle weight $Z_{\Lambda}(\mathbf{p})$ is always close to unity for the attractive HM, higher-order quantum fluctuations do not seem to be so crucial in this case. This should be contrasted with the repulsive HM, where quantum fluctuations are generally believed to be stronger and, consequently, of central importance to describe its low-energy dynamics.

\section{Acknowledgements}

Two of us (EC and HF) want to thank the International Institute of Physics for the kind hospitality. One of us (AF) acknowledge support by CNPq, FINEP, and MCT (Brazil).

\section{References}

1. For a recent review, see e.g., I. Bloch, J. Dalibard and W. Zwerger, Rev. Mod. Phys. 80, 885 (2008).

2. Th. Maier et al., Phys. Rev. Lett. 85, 1524 (2000).

3. T. A. Maier et al., Phys. Rev. Lett. 95, 237001 (2005). 
4. B. Kyung et al., Phys. Rev. B 73, 165114 (2006).

5. L. Arrachea and D. Zanchi, Phys. Rev. B 71, 064519 (2005).

6. D. Zanchi and H. J. Schulz, Phys. Rev. B 54, 9509 (1996).

7. D. Zanchi and H. J. Schulz, Phys. Rev. B 61, 13609 (2000).

8. C. J. Halboth and W. Metzner, Phys. Rev. B 61, 7364 (2000).

9. C. J. Halboth and W. Metzner, Phys. Rev. Lett. 85, 5162 (2000).

10. C. Honerkamp et al., Phys. Rev. B 63, 035109 (2001).

11. R. Shankar, Rev. Mod. Phys. 66, 129 (1994).

12. P. Kopietz and T. Busche, Phys. Rev. B 64, 155101 (2001).

13. S. W. Tsai and J. B. Marston, Can. J. Phys. 79, 1463 (2001).

14. J. Gonzalez, F. Guinea and M. A. H. Vozmediano, Phys. Rev. Lett. 79, 3514 (1997).

15. B. Binz, D. Baeriswyl and B. Doucot, Eur. Phys. J. B 25, 69 (2002).

16. A. Ferraz, Phys. Rev. B 68, 075115 (2003).

17. H. Freire, E. Correa and A. Ferraz, Phys. Rev. B 71, 165113 (2005).

18. F. Wegner, Ann. Phys. Leipzig 3, 77 (1994).

19. I. E. Dzyaloshinsky and I. A. Larkin, Sov. Phys. - JETP 38, 202 (1974).

20. J. Solyom, Adv. Phys. 28, 201 (1979).

21. For a review, see J. Voit, Rep. Prog. Phys. 58, 977 (1995).

22. A. Ferraz, Phys. Rev. B 75, 233103 (2007).

23. A. Ferraz, J. Phys. A: Math. Gen. 39, 7963 (2006).

24. H. Freire, E. Correa and A. Ferraz, Phys. Rev. B 78, 125114 (2008).

25. E. Correa, H. Freire and A. Ferraz, Phys. Rev. B 78, 195108 (2008).

26. R. Micnas, J. Ranninger and S. Robaszkiewicz, Rev. Mod. Phys. 62, 113 (1990).

27. R. Gersch, C. Honerkamp and W. Metzner, New J. Phys. 10, 045003 (2008).

28. R. T. Scalettar et al., Phys. Rev. Lett. 62, 1407 (1989).

29. J. E. Hirch and D. J. Scalapino, Phys. Rev. Lett. 56, 2732 (1986).

30. A. Moreo and D. J. Scalapino, Phys. Rev. Lett. 66, 946 (1991).

31. Th. Paiva et al., Phys. Rev. B 69, 184501 (2004).

32. J. M. Singer, T. Schneider and M. H. Pedersen, Eur. Phys. J. B 2, 17 (1998).

33. J. M. Singer, T. Schneider and P. F. Meier, Eur. Phys. J. B 7, 37 (1999).

34. M. Keller, W. Metzner and U. Schollwöck, Phys. Rev. Lett. 86, 4612 (2001).

35. M. Keller, W. Metzner and U. Schollwöck, J. Low Temp. Phys. 126, 961 (2002).

36. M. Capone, C. Castellani and M. Grilli, Phys. Rev. Lett. 88, 126403 (2002).

37. D. Rohe and W. Metzner, Phys. Rev. B 63, 224509 (2001).

38. N. Trivedi and M. Randeria, Phys. Rev. Lett. 75, 312 (1995).

39. B. Kyung, S. Allen and A.-M. S. Tremblay, Phys. Rev. B 64, 075116 (2001).

40. A good review on the BCS treatment of the attractive Hubbard model can be found in T. Schneider and J. M. Singer, Phase Transition Approach To High Temperature Superconductivity (Imperial College Press, London, 2000).

41. A. Martín-Rodero and F. Flores, Phys. Rev. B 45, 13008 (1992). 